\title{
Pulmonary arterial disease with multifocal dissecting haemorrhage in a newborn infant
}

\author{
P. V. BEST AND D. HEATH \\ From the Departments of Pathology, Universities of Aberdeen and Birmingham
}

Intramural haemorrhage in pulmonary arteries is very rare and usually takes the form of a dissecting aneurysm in the pulmonary trunk or a major pulmonary artery. It usually develops on the basis of cystic medial necrosis and pulmonary hypertension. We report a newborn infant with congenital heart disease in whom multifocal haemorrhage occurred in the media of elastic and muscular pulmonary arteries weakened by a structural abnormality, which, so far as we know, has not been described before. A similar, but not identical, lesion has recently been reported by van Unnik (1963, 1964).

\section{CASE REPORT}

A female infant born on 3 January 1963 was admitted to hospital 16 days later on account of cyanotic attacks. The mother's age was 31 years and there was one other daughter aged 3 years, who was healthy. During the sixth week of pregnancy the mother had had abdominal pain and sickness, and later the pregnancy had been complicated by hydramnios. Although the expected date of delivery was 18 December 1962, the infant weighed only $4 \mathrm{lb} .8 \mathrm{oz}$. at birth: delivery was spontaneous and uncomplicated, and lasted four hours.

The condition of the baby at birth was satisfactory, but cyanosis and difficulty with feeding developed and she was admitted to hospital. On examination she was noted to have an odd facial appearance with low-set pinnae, both abnormally shaped, especially the left, which was curled and 'shell-shaped'. There was a high, arched palate. The eyes were narrow, giving a somewhat mongoloid appearance, the head was small, and the forehead was bossed. The left wrist and the fingers of both hands, especially the left, were held in flexion. The left thumb was a small rudimentary structure, and the hallux on both sides was disproportionately small. The skin had a slightly dusky tinge but was not frankly cyanosed. The infant's temperature on admission was recorded as $96.8^{\circ} \mathrm{F}$. but rose to normal levels in a few hours. The heart rate was 132 per minute and the pulse was regular. The systolic blood pressure was $130 \mathrm{~mm}$. Hg but the diastolic pressure could not be recorded. A blowing systolic murmur was heard in most areas, especially the aortic area, where the second sound was loud, but diastolic murmurs were not heard? Crepitations were present at the right lung base, and the liver was slightly enlarged.

A radiograph of the chest showed partial collapses at the right base with displacement of the heart to that side. Features of right ventricular hypertrophy were seen on the electrocardiogram.

The infant became extremely cyanosed shortly afte admission, and she was thereafter nursed in an oxygentent: cyanosis persisted, her condition rapidlyo deteriorated, and she died on the following day when 17 days old.

NECROPSY The heart weighed 25 g. (see Fig. 1). Th superior and inferior venae cavae were normal. Therहू was a small, functionally patent foramen ovale bug no abnormal atrial septal defect. The right atrium was dilated and hypertrophied. The tricuspid valve was a wide, dilated orifice which did not show three separate cusps, but was in the form of a single cur tain: its circumference was $4 \mathrm{~cm}$. The right ventricle. was dilated and hypertrophied, the thickness of the ventricular wall being 6 to $8 \mathrm{~mm}$. A narrow ventricula? septal defect, $5 \mathrm{~mm}$. in diameter, was situated in the uppermost, membranous portion of the ventriculap septum. The pulmonary valve was tricuspid and. appeared normal. The pulmonary trunk divided int two large pulmonary arteries which passed normally to the lungs. A large 'window-type' patent ductus arteriosus connected the origin of the left main pul monary artery with the arch of the aorta. The walls of the main pulmonary arteries and their branches in. the hilum of each lung appeared to be thicker thaff normal, but there were no other macroscopic abnormalities in the lungs. The pulmonary veins wers normal and entered at the usual sites in the left atrium, which showed no abnormality. The mitrat valve was atretic, so that even a fine probe could nof be passed through it into the diminutive left ventricle whose wall was only $3 \mathrm{~mm}$. thick. Since a probe could not be passed through the aortic valve, it too was assumed to be atretic. Two coronary arteries arose from the region of origin of the ascending aorta Their distribution was normal. The ascending aort was hypoplastic, its size barely exceeding that of th 8 coronary arteries. However, at the junction of the ascending portion and the arch, at the point of entry 


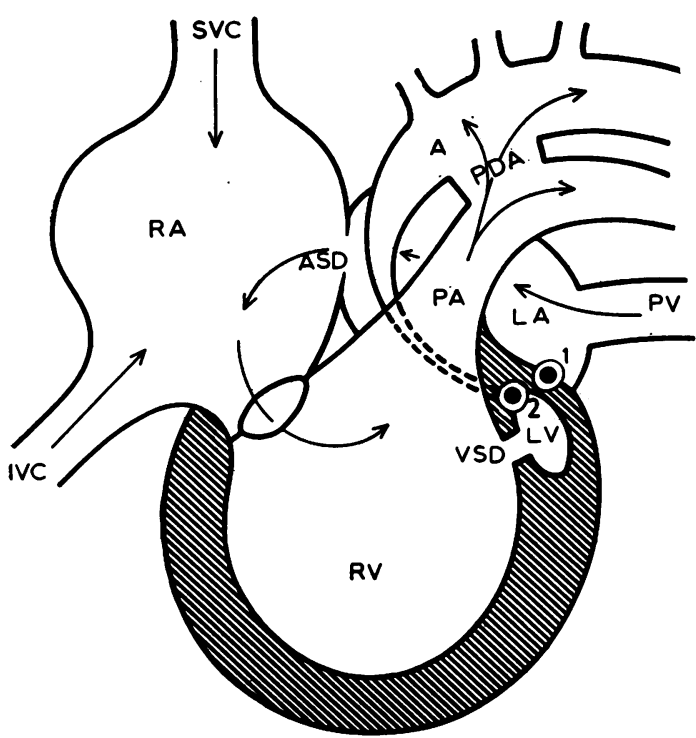

FIG. 1. Diagram of the heart to show the congenital abnormalities and the direction of intracardiac blood flow. $S V C$, superior vena cava; IVC, inferior vena cava; $A S D$, atrial septal defect; $R V$, right ventricle; VSD, ventricular septal defect; $L V$, left ventricle; $P A$, pulmonary artery; $P D A$, patent ductus arteriosus; $A$, aorta; $P V$, pulmonary vein; LA, left atrium; 1, atretic mitral valve; 2 , atretic aortic valve. The dotted lines represent the hypoplastic ascending aorta.

[After Edwards, J. E. In Pathology of the Heart, Gould, S. E. Charles C. Thomas, Springfield, Illinois.]

of the wide patent ductus arteriosus from the pulmonary circulation, the aorta showed abrupt widening, so that its diameter and thickness became normal. The abdominal viscera and brain showed no notable abnormalities.

DIAGNOSIS Mitral atresia ; aortic atresia ; ventricular septal defect; and patent ductus arteriosus.

HISTOLOGY Abnormalities were present in all classes of pulmonary arteries, but they were most pronounced in the elastic pulmonary arteries. In many of these the outer half of the media was enormously expanded and the components of the media were unusually loosely packed together (Fig. 2). Fibroblasts, formed collagen fibres, and fusiform smooth muscle cells were identifiable by their characteristic tinctorial properties with Van Gieson's stain. The individual cellular elements were widely separated from one another. This enormous expansion and looseness of the outer media was associated with degeneration and disruption of the elastic laminae (Fig. 3). Usually in the foetal elastic pulmonary arteries the elastic laminae are uniform and unbranched and form evenly-spaced concentric rings, but in this infant the elastic laminae were often so widely separated that they formed a mosaic pattern (Fig. 4). The outer elastic laminae appeared to have been forced out by the swelling of the outer media, so that the contour of the periphery of the arteries was tuberose rather than regular. Many of the elastic laminae appeared abnormally thick, and some of them showed degeneration of elastin with alternate bands of normal and decreased density. In some elastic fibrils this degeneration had progressed to frank rupture, so that frayed ends of some of them ended abruptly in the loose tissues of the outer media (Fig. 3). In some places there were ruptures in the internal elastic lamina (Figs 3 and 5). Some of the elastic fibrils showed numerous fenestrations along their length, and in many instances the radial connexions of damaged elastic laminae were still intact. In contrast, the elastic fibrils of the inner half of the media were abnormally tightly packed together and were parallel with one another (Figs 2 and 4). Thus the appearances of the elastica of the inner and outer halves of the media formed a striking contrast to one another.

Extensive haemorrhage had occurred into the loose tissues of the outer media (Fig. 4). This bleeding was interstitial and appeared to have produced part of the separation of fibrous tissue and smooth muscle cells described above. In many cases the bleeding was pronounced, forming lakes of blood in the outer media. The haemorrhage usually permeated into the surrounding loose tissues without an intervening structural barrier (Fig. 4), but in a few instances there was a distinct endothelial lining to give the appearance of fragile sinus-like vessels up to $1.5 \mathrm{~mm}$. in diameter in the outer media (Figs. 5, 6, and 7). No connexions of well-defined structure between these sinuses and either the lumen of the vessel or the periarterial space could be demonstrated in serial sections. Ill-defined interstitial haemorrhages extended from the margins of these lakes of blood to the internal elastic lamina and to the adventitia. In some arterial segments the haemorrhage in the outer part of the media was so severe that it displaced the inner layer and compressed the lumen, which was occasionally reduced to a narrow slit (Fig. 8). The intima of the pulmonary arteries was also abnormal, showing extensive plaques of thickening due to fibroelastosis (Figs 2 and 4). In some vessels the thickness of these intimal plaques was half that of the underlying media. In many sites there was bleeding into peri-arterial tissues. Thus artefactual peri-arterial spaces had been brought about by such peri-arterial bleeding. There were, however, also haemorrhages into true peri-arterial lymphatics readily identified by their paired valves. Deposition of haemosiderin in the peri-arterial connective tissue was a notable feature.

Although these abnormalities were most pronounced in the elastic pulmonary arteries, the looseness of the tissues of the outer media, without associated haemorrhage, was also found in a few small interlobular and intralobular pulmonary arteries (Fig. 9). 


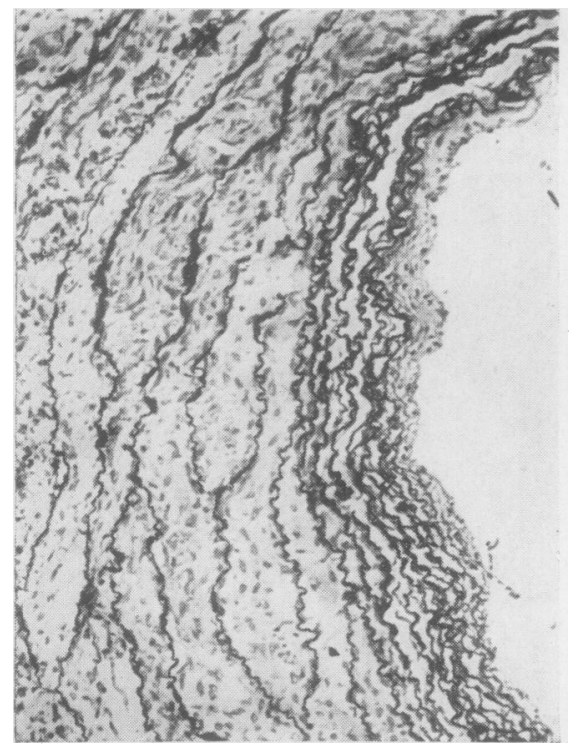
tightly together. Those of the outer media are widely separated by loose fibrous tissue and smooth muscle fibres. There is intimal fibroelastosis. $\times 100$.

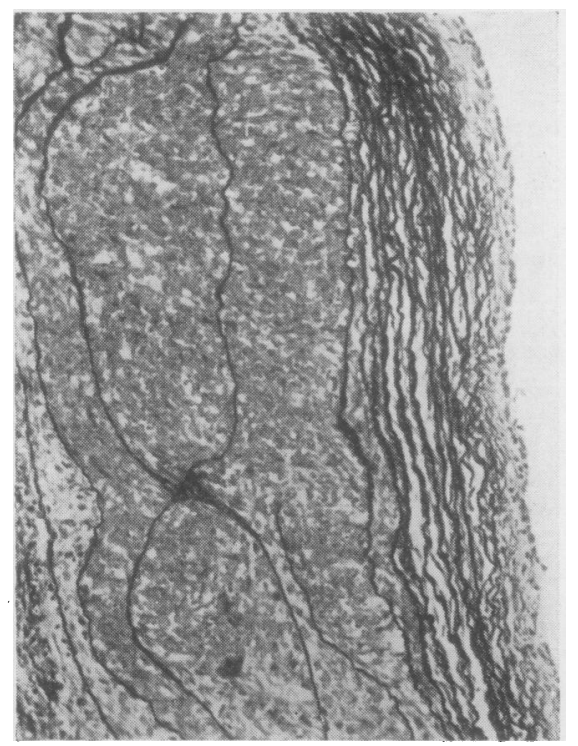

FIG. 4. The elastic laminae of the inner media are tightly packed and parallel. Those of the outer media are widely separated into a mosaic pattern. Interstitial haemorrhage has taken place into the loose tissues of the media. $\times 100$.

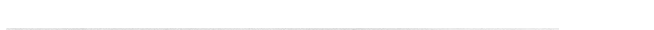
$412 \quad$ P. V. Best and D. Heath

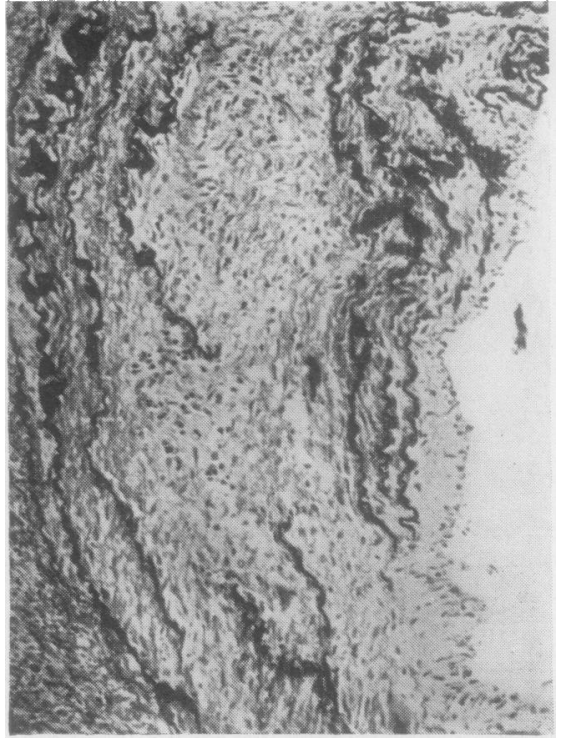
the free ends of which end in the abnormally loose fibro-muscular tissues of the media. There is also rupture of the internal elastic lamina. $\times 100$.

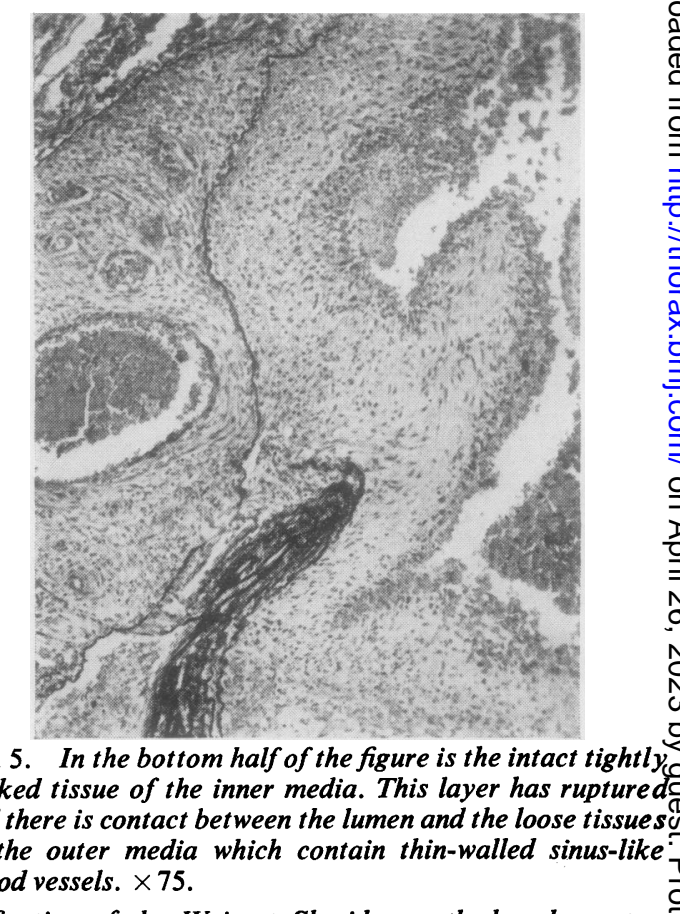

FIG. 5. In the bottom half of the figure is the intact tightly packed tissue of the inner media. This layer has ruptured and there is contact between the lumen and the loose tissuese of the outer media which contain thin-walled sinus-like blood vessels. $\times 75$. P. V. Best and D. Heath

All the sections are stained for elastic by the Lawson modification of the Weigert-Sheridan method and counter-市

stained with Van Gieson's stain. 2 to 5 are segments of the wall of elastic pulmonary arteries. In each case the lumen and inner part of the media is to the right. 


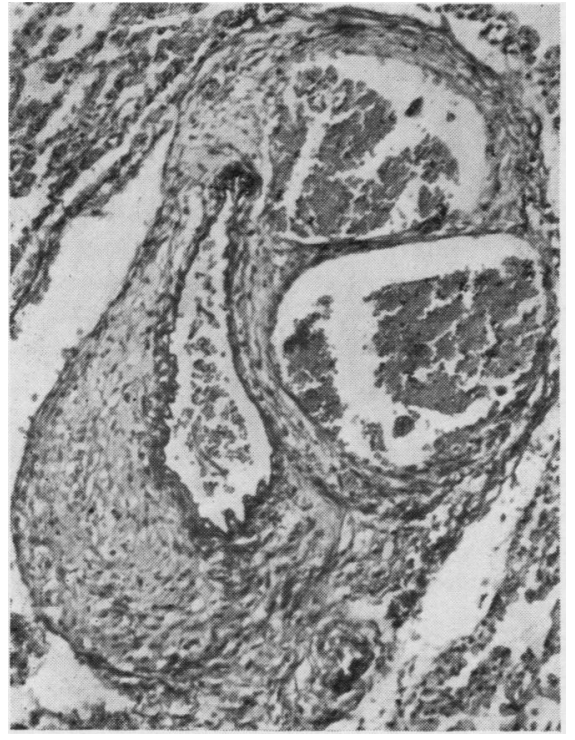

FIG. 6. Distended, thin-walled blood vessels are present in the loose tissues of the outer media pushing the inner media into the lumen. $\times 100$.

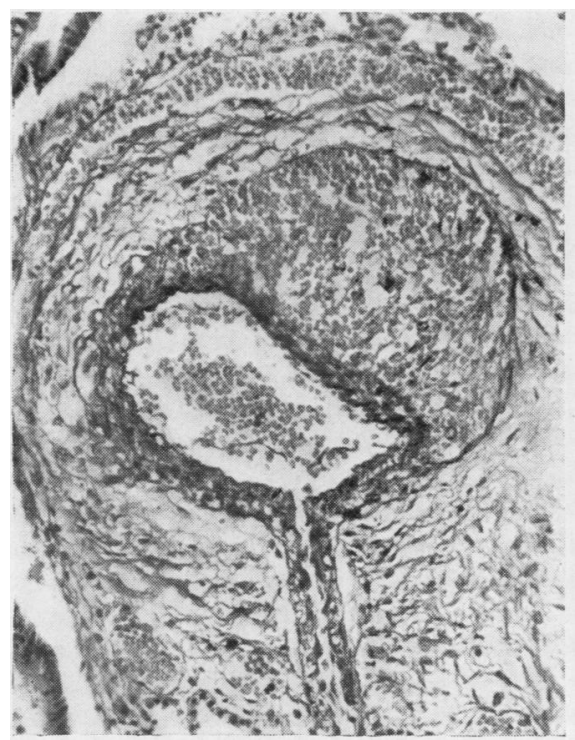

FIG. 7. Distended, thin-walled blood vessel in the loose tissues of the outer media which has pushed the inner media into the lumen. A thick-walled arteriolar branch arises from the lower part. $\times 85$.

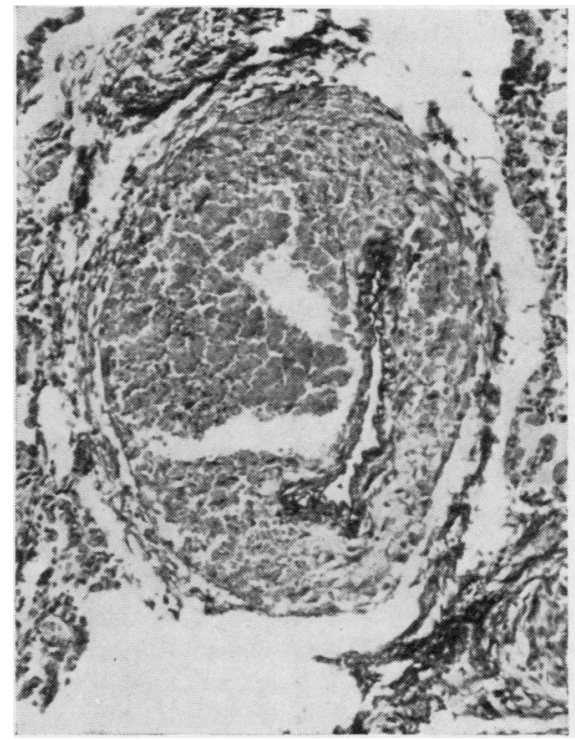

FIG. 8. Haemorrhage into the loose tissues of the outer media compresses the inner media, reducing the lumen to a mere slit. $\times 100$.

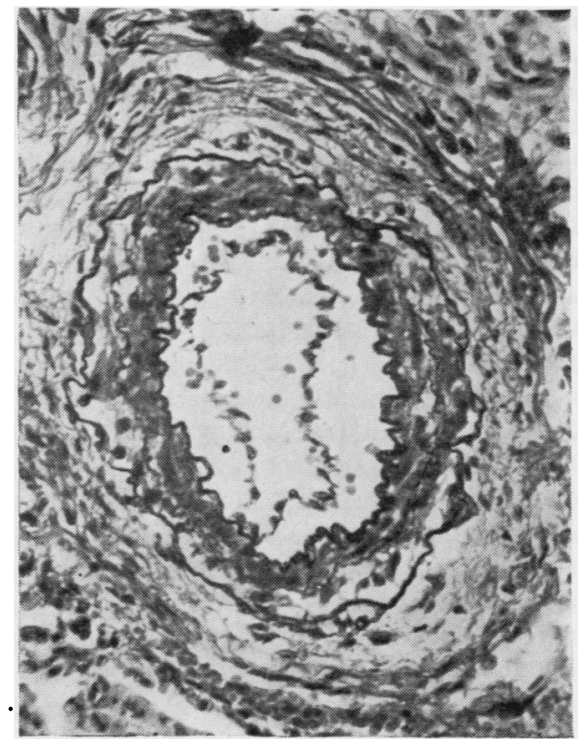

FIG. 9. The inner media shows the normal compact arrangement of smooth muscle fibres. The outer media is abnormally loose and composed of fibrous tissue and widely separated smooth muscle fibres. $\times 215$.

Figs 6 to 9 are transverse sections of interlobular pulmonary arteries. 
The effect of this was to make the thickness of the media relative to the external diameter of the vessel even more pronounced than in the normal newborn infant. In intralobular arteries of $30 \mu$ external diameter the medial thickness was $25 \%$. Such vessels had a prominent endothelial lining and a thick adventitia. The pulmonary veins and venules and the bronchial arteries were normal. So, too, were some of the elastic pulmonary arteries which had distinct internal and external elastic laminae and about six concentric elastic laminae. In sections of lung stained with alcian blue there were no macrophages filled with acid mucopolysaccharides in the walls of the pulmonary arteries: we looked especially for this because of the possibility of gargoylism. The aorta and the systemic arteries appeared normal.

\section{DISCUSSION}

So far as we are aware there are no previous reports of a pulmonary arterial disease of the type described here, which consists of multifocal haemorrhage into media of abnormal thickness and loose structure. The presence of haemosiderin in the peri-arterial connective tissues indicates that the bleeding started at least a few days before the infant died.

In view of the odd external appearance of the body, we considered the possibility that the pulmonary vascular changes were those rarely associated with Hurler's syndrome or gargoylism (see McKusick, 1960). In this inborn error of metabolism there is an excessive production of acid mucopolysaccharides, which may be ingested by macrophages in the media and intima of pulmonary arteries (Wagenvoort, Heath, and Edwards, 1964). In the present case such substances were not demonstrable in the pulmonary vessels, and the condition is not normally diagnosable from the external appearance of the body before the child is about 6 months old.

The commonest cause of intramural haemorrhage in pulmonary arteries is a dissecting aneurysm of one of the large conducting pulmonary arteries, but even this is very rare. In his review of dissecting aneurysms of arteries other than the aorta, Watson (1956) could find reports of only four cases involving the pulmonary arteries. Since that review there have been a few further isolated reports such as those of Fleming (1956), Foord and Lewis (1959), and Ravines (1960). Such cases are, however, rather different from that described here because, in general, dissection has occurred in the major elastic pulmonary arteries either within or outside the lung. Most of the affected patients have been adults with heart disease, usually congenital, and a long history of pulmonary arterial hypertension associated in some cases with cystic medial necrosis of the elastic pulmonary arteries. It is easy to see how dissection could occur in a media rendered abnormally fragile by accumulation of excess acid mucopolysaccharides at the very time when it is subjected to the added stress of a raised pulmonary arterial pressure.

The present case has certain similarities in that congenital heart disease was present and was certainly associated with a high pulmonary arterial pressure. Since the pulmonary arteries and the aorta were in direct communication by means of a wide patent ductus arteriosus, the blood pressure in the lesser and systemic circulations must have been very similar. The media also showed an abnormality of structure which must have made it more fragile than normal. Here the similarities end, for the patient was a newborn infant, and the increased fragility of the media was due not to an excess of acid mucopolysaccharides but to an abnormal, loose structure. As a result of this the haemorrhages were multifocal rather than single.

The nature of the abnormality of the media is not clear. We know that the type of heart disease present in this case is not always associated with this peculiar vascular lesion, since we have recently found normal pulmonary arteries in a female infant aged 1 week with identical congenital cardiac anomalies. Although there is a variable individual susceptibility to the effects of pulmonary hypertension, this suggests to us that the abnormalities in the media do not depend primarily on the altered pulmonary haemodynamics brought about by this congenital abnormality of the heart. Furthermore, in our previous studies of the various forms of hypertensive pulmonary vascular disease we have never seen a vascular lesion of this type due primarily to pulmonary hypertension. This observation, however, does not exclude the possibility that in the present case pulmonary hypertension further damaged the already abnormal and fragile pulmonary arteries, leading to multiple dissecting haemorrhages within their walls.

We have recently been privileged to examine with Dr. van Unnik of Rotterdam some sections of lungs from six of his cases showing similar but not identical pulmonary vascular lesions. There were what he calls 'dissecting haemorrhages' into the media of elastic and muscular pulmonary arteries bringing about disruption and separation of the components of the media. There was also haemorrhage into peri-arterial spaces and true peri-arterial lymphatics. Such histological appearances are similar to those described here. van 
Unnik (1964) believes the intramural pulmonary vascular haemorrhages in some of his cases to be the result of hypothermia due to neonatal exposure to cold. He points out that such neonatal cold injury may lead to bleeding into alveoli, bronchi, and pulmonary interstitial tissues as well as into the walls of pulmonary arteries. Pulmonary hypertension does not play an important role in the aetiology of the condition. His other cases had infective conditions such as staphylococcal pneumonia or Salmonella infection, but in these the dissecting haemorrhages occurred only in small muscular pulmonary arteries.

The vascular disease which we report here differs from that described by van Unnik in one important respect. In the present case the pulmonary arteries were structurally abnormal and not merely disrupted by intramural haemorrhage. Furthermore, although the body temperature of this patient was slightly low on admission it rose to normal levels in a few hours. It is conceivable that the form of pulmonary vascular disease described in this paper is merely a more chronic stage of the lesion reported by van Unnik and represents organization of intramural haemorrhage produced primarily by hypothermia or some other unknown factor. The structural state of the pulmonary arteries and the presence of haemosiderin in our case leads us to believe that the pulmonary vasculature was already abnormal in intra-uterine life. The survival of the patient to the age of 17 days, however, is consistent with a primary intramural haemorrhage which occurred in the neonatal period, due to some cause unknown to us, and which gave rise to the other changes described above by initial disruption of the components of the media.

Nevertheless the possibility remains that the pulmonary vascular disease present in this infant might be a hitherto undescribed congenital abnormality of the pulmonary vasculature, and that the haemorrhages were produced by the effects of pulmonary hypertension on the media rendered abnormally fragile by its maldevelopment.

\section{SUMMARY}

A female infant with combined aortic and mitral atresia associated with a ventricular septal defect and patent ductus arteriosus died 17 days after birth. Many of the elastic and muscular pulmonary arteries in both lungs were grossly abnormal in that there were multifocal haemorrhages into the media, the outer half of which was greatly expanded by an unusual looseness and separation of its components. The nature of this vascular disease is obscure. It may be a chronic stage of intramural haemorrhage brought about by exposure to cold or infection. It may be a congenital abnormality of the pulmonary vasculature. The disorder is unlikely to be due to pulmonary hypertension alone, but elevation of arterial pressure may increase the damage brought about by other factors.

We wish to thank Dr. N. S. Clark for allowing us to report this case: we are grateful to Dr. J. A. M. van Unnik and Dr. Jesse E. Edwards for discussing certain aspects of it with us.

\section{REFERENCES}

Fleming, H. A. (1956). Aorto-pulmonary septal defect with patent ductus arteriosus and death due to rupture of dissecting aneurysm of the pulmonary artery into the pericardium. Thorax, 11, 71 .

Foord, A. G., and Lewis, R. D. (1959). Primary dissecting aneurysms of peripheral and pulmonary arteries. Arch. Path., 68, 553.

McKusick, V. A. (1960). The Hurler Syndrome. Chapter 7 in Heritable Disorders of Connective Tissue, p. 242, 2nd ed. C. V. Mosby, St. Louis.

Ravines, H. T. (1960). Dissecting hematomas of intrapulmonary arteries in a case of pulmonary hypertension associated with patent ductus arteriosus. J. thorac. cardiovasc. Surg., 39, 760.

van Unnik, J. A. M. (1963). Afwijkingen van de longarteriën bij zuigelingen na accidentele hypothermie. (Lesions of pulmonary arteries in infants after accidental hypothermia.) Ned. T. Geneesk., $107,1069$.

- (1964). Dissecting haemorrhage in medium sized pulmonary arteries in infants. Paper at joint meeting of Dutch and British Pathological Societies, Groningen, 1964.

Wagenvoort, C. A., Heath, D., and Edwards, J. E. (1964). The Pathology of the Pulmonary Vasculature. Charles C. Thomas,

Watson, A.J. (1956). Dissecting aneurysm of arteries other than the aorta. J. Path. Bact., 72, 439. 